\title{
Carbohydrate, dietary glycaemic index and glycaemic load, and colorectal cancer risk: a case-control study in China
}

\author{
Jing Huang ${ }^{1,2}$, Yu-Jing Fang ${ }^{3,4}$, Ming Xu ${ }^{1}$, Hong Luo ${ }^{1}$, Nai-Qi Zhang ${ }^{1}$, Wu-Qing Huang ${ }^{1}$, Zhi-Zhong Pan ${ }^{3}$, \\ Yu-Ming Chen ${ }^{1,2}$ and Cai-Xia Zhang ${ }^{1,2 *}$ \\ ${ }^{1}$ Department of Medical Statistics and Epidemiology, School of Public Health, Sun Yat-sen University, Guangzhou 510080, \\ People's Republic of China \\ ${ }^{2}$ Guangdong Provincial Key Laboratory of Food, Nutrition and Health, School of Public Health, Sun Yat-sen University, \\ Guangzhou 510080, People's Republic of China \\ ${ }^{3}$ State Key Laboratory of Oncology in South China, Department of Colorectal Surgery, Sun Yat-sen University Cancer \\ Center, Collaborative Innovation Center for Cancer Medicine, 651 Dongfeng Road East, Guangzhou 510060, People's \\ Republic of China \\ ${ }^{4}$ State Key Laboratory of Oncology in South China, Department of Experimental Research, Sun Yat-sen University \\ Cancer Center, Collaborative Innovation Center for Cancer Medicine, 651 Dongfeng Road East, Guangzhou 510060, \\ People's Republic of China
}

(Submitted 21 September 2017 - Final revision received 10 January 2018 - Accepted 2 February 2018)

\section{Abstract}

A carbohydrate-rich diet results in hyperglycaemia and hyperinsulinaemia; it may further induce the carcinogenesis of colorectal cancer. However, epidemiological evidence among Chinese population is quite limited. The aim of this study was to investigate total carbohydrate, non-fibre carbohydrate, total fibre, starch, dietary glycaemic index (GI) and glycaemic load (GL) in relation to colorectal cancer risk in Chinese population. A case-control study was conducted from July 2010 to April 2017, recruiting 1944 eligible colorectal cancer cases and 2027 age (5-year interval) and sex frequency-matched controls. Dietary information was collected by using a validated FFQ. The OR and 95\% CI of colorectal cancer risk were assessed by multivariable logistic regression models. There was no clear association between total carbohydrate intake and colorectal cancer risk. The adjusted OR was 0.85 (95\% CI 0.70, 1.03, $\left.P_{\text {trend }}=0.08\right)$ comparing the highest with the lowest quartile. Total fibre was related to a $53 \%$ reduction in colorectal cancer risk (adjusted OR $\mathrm{OR}_{\text {quartile }} 4 v .10 \cdot 47 ; 95 \%$ CI $0 \cdot 39$, 0.58). However, dietary GI was positively associated with colorectal cancer risk, with an adjusted $\mathrm{OR}_{\text {quartile } 4 v .1}$ of 3.10 (95\% CI 2.51, 3.85). No significant association was found between the intakes of non-fibre carbohydrate, starch and dietary GL and colorectal cancer risk. This study indicated that dietary GI was positively associated with colorectal cancer risk, but no evidence supported that total carbohydrate, non-fibre carbohydrate, starch or high dietary GL intake were related to an increased risk of colorectal cancer in a Chinese population.

Key words: Carbohydrates: Glycaemic index: Glycaemic load: Colorectal cancer: Case-control studies

There were 1.4 million colorectal cancer cases and 693900 deaths caused by this disease worldwide in $2012^{(1)}$. In China, the incidence of colorectal cancer risk presents an increasing trend and has become the fourth common cancer, with an estimated 376300 cases occurring in $2015^{(2)}$. The epidemiological evidence supported that dietary and lifestyle factors may play a pivotal role in the aetiology of colorectal cancer risk ${ }^{(3)}$. However, on the basis of the current available evidence, only alcohol consumption and red and processed meat are the firm causes of colorectal cancer risk ${ }^{(4)}$. Thus, the association between other dietary factors and colorectal cancer risk needs more epidemiological studies to confirm.
It was reported that diabetes was related to an increased risk of colorectal cancer ${ }^{(5,6)}$. The most important mechanism underlying the carcinogenesis was hyperglycaemia, hyperinsulinaemia or inflammation ${ }^{(7)}$. Insulin could enhance the concentration of insulin-like growth factor (IGF) by decreasing the level of IGF-binding proteins ${ }^{(8)}$, and IGF play a vital role in cell growth and differentiation ${ }^{(9)}$. Carbohydrate is the main dietary component affecting blood glucose and insulin concentrations $^{(10)}$. Carbohydrate-rich diet results in hyperglycaemia, and then hyperinsulinaemia; thus, it may further induce the carcinogenesis of colorectal cancer. However, the effect of carbohydrate on blood glucose may depend on the

Abbreviations: GI, glycaemic index; GL, glycaemic load; IGF, insulin-like growth factor; MET, metabolic equivalent task.

* Corresponding author: Professor C.-X. Zhang, fax +86 20 87330446, email zhangcx3@mail.sysu.edu.cn 
type and amount of carbohydrate consumed. For example, fibre cannot be absorbed in the intestine, whereas the non-fibre carbohydrate, including starch, can be absorbed in the small intestine and lead to an increase of blood glucose ${ }^{(11)}$. Glycaemic index (GI) of foods is an index for ranking their carbohydrate content based on their postprandial blood glucose effects, which is defined as the area under the 2-h blood glucose response curve, divided by the corresponding area of the same amount of carbohydrate from a standard reference (glucose or white bread) ${ }^{(12)}$. Glycaemic load (GL) is an index further considering the quantity of carbohydrate consumed, which is calculated as the product of the GI value and the amount of available carbohydrate, and dividing the total by $100^{(13)}$. Therefore, GL is a comprehensive measure of both the blood glucose effects (quality) of the food and the total quantity of carbohydrate consumed ${ }^{(14)}$.

Some observational studies examined the association between dietary carbohydrate, GI and GL and colorectal cancer risk, but failed to draw a consistent conclusion. A cohort study found that high dietary GI and carbohydrate intake from highGI foods, but not GL, were associated with an increased risk of colorectal cancer ${ }^{(14)}$. Similarly, a meta-analysis analysing eight cohort studies and five case-control studies found that dietary GI was related to an increased risk of colon cancer ${ }^{(15)}$. Another meta-analysis considering only cohort studies also revealed that dietary GI had a borderline positive relationship with colorectal cancer risk ${ }^{(16)}$. However, one meta-analysis including fourteen cohort studies concluded that there was no clear association between dietary carbohydrate, GI and GL and the risk of colorectal cancer ${ }^{(11)}$. Regarding the different types of carbohydrate, a meta-analysis including sixteen studies revealed that total dietary fibre intake was related to a reduced risk of colorectal cancer ${ }^{(17)}$. Some studies focused on dietary non-fibre carbohydrate intake. One cohort study found no clear association between non-fibre carbohydrate intake and colorectal cancer risk ${ }^{(14)}$. However, another cohort study ${ }^{(18)}$ and one case-control study ${ }^{(19)}$ observed that consumption of non-fibre carbohydrate was associated with an increased risk of colorectal cancer. So far, two case-control studies providing inconsistent conclusions have been conducted to examine the association between starch intake and colorectal cancer risk ${ }^{(20,21)}$. To date, only one relevant study has been conducted among Chinese population, yet it focused on only women ${ }^{(22)}$.

This case-control study aimed to evaluate the association between dietary intake of total carbohydrate, non-fibre carbohydrate, total fibre, starch, GI and GL and colorectal cancer risk. We hypothesised that diets high in total carbohydrate, non-fibre carbohydrate, starch, dietary GI and GL were associated with increased colorectal cancer risk, whereas high intake of total fibre was inversely associated with colorectal cancer risk.

\section{Methods \\ Study subjects}

This is an ongoing case-control study that began in July 2010, and the study design has been described previously ${ }^{(23)}$. In brief, potential case subjects were consecutively recruited from
Sun Yat-sen University Cancer Center, Guangzhou, China, from July 2010 to April 2017. Eligible patients were aged 30-75 years and natives of Guangdong province or had lived in Guangdong for at least 5 years, with histologically confirmed colorectal cancer diagnosed no more than 3 months. Patients were excluded if they could not understand or speak Mandarin/ Cantonese or had a prior history of any cancer. A total of 2230 eligible cases were identified and 1961 were successfully interviewed. In all, 269 patients failed to complete the investigation, mainly because of fatigue, communication barriers and refusal. Thus, the response rate was $87.93 \%$. A total of seventeen subjects with an extreme energy intake $(<2510$ or $>14644 \mathrm{~kJ} / \mathrm{d}(<600$ or $>3500 \mathrm{kcal} / \mathrm{d})$ for women, $<3347$ or $>17573 \mathrm{~kJ} / \mathrm{d}$ ( $<800$ or $>4200 \mathrm{kcal} / \mathrm{d}$ ) for men) were excluded in the analysis. Finally, 1944 cases were included in the analysis.

Two control groups were used in this study, which were frequency matched by age (5-year internal) and sex to the case patients. The first control group was recruited from Departments of Vascular Surgery, Otorhinolaryngology and Plastic and Reconstructive Surgery in the First Affiliated Hospital of Sun Yat-sen University during the same time period as the cases. They mainly suffered from chronic otitis media, chronic sinusitis, sudden deafness, vocal cord polyp, trigeminal neuralgia, varicose veins, orthopaedics and facial paralysis. The second control group was recruited from residents in the same community via community advertisements, written invitations or subjects' referrals. The same inclusion and exclusion criteria that were used to the cases were applied to the controls, except that they had no prior history of colorectal cancer. Totally, 1327 hospital-derived controls were identified and 1168 were successfully interviewed, with a participation rate of $88.02 \%$. In addition, 859 community-derived controls were interviewed. The total number of controls was 2027.

We assumed that there were $25 \%$ people with higher carbohydrate intake, GI and GL among the general population, and the estimated OR between carbohydrate intake, GI and GL and colorectal cancer risk was $1.51,1.35$ and $1.43^{(14)}$, respectively, the type I error rate was $<0.05(\alpha=0.05)$, the power of test was $90 \%(\beta=0 \cdot 10)$ and the response rate was $80 \%$. On the basis of these assumptions, we required a sample size of 755 cases for carbohydrate, 1454 cases for GI and 1013 cases for GL.

This study was conducted according to the Declaration of Helsinki. The Ethical Committee of School of Public Health, Sun Yat-sen University, approved all procedures. Written informed consent was obtained from all participants before the interview.

\section{Data collection}

All study subjects were interviewed face-to-face by trained interviewers using a structured questionnaire, in order to collect information on socio-demographic characteristics, lifestyle factors (e.g. active smoking, passive smoking, alcohol drinking and physical activity), family history of cancer and body measurements (weight and height). The body weight was measured and the height was self-reported. BMI was calculated as the ratio of weight $(\mathrm{kg})$ to squared height $\left(\mathrm{m}^{2}\right)$. The diagnosis and histological findings were collected from the medical records. The study subjects were also asked to answer the 
question "Have you had physician-diagnosed diabetes mellitus?'. For women, menstrual conditions and reproductive experiences were also obtained. In this study, active smokers were defined as people who smoked at least 1 cigarette/d for more than 6 months consecutively or accumulatively in their lifetime ${ }^{(24)}$. Passive smokers were defined as non-smokers who reported being exposed to the smoke exhaled by smokers for at least $15 \mathrm{~min} / \mathrm{d}$ over a week. Regular drinking was defined as drinking alcohol at least once per week over the previous year. The intensity of physical activity was assessed based on self-reported occupational, household and leisure activity in the past year. Information on frequency $(\mathrm{d} /$ week) and duration $(\mathrm{h} / \mathrm{d})$ for household and leisure physical activities were obtained. According to labour intensity, occupational activity was classified as follows: (a) not working; (b) sitting for a long time; (c) low intensity; (d) moderate intensity; or (e) vigorous intensity, with examples provided. Household and entertainment activities were combined in this study. They were categorised into light physical activity (e.g. walking), moderate physical activity (e.g. jogging, mountaineering, playing table tennis) and vigorous physical activity (e.g. running, playing football/basketball). The mean metabolic equivalent task-hours (MET) value of each physical activity was acquired by estimating the average of all comparable activities in the Compendium of Physical Activities ${ }^{(25,26)}$. MET-h/week over the past one year was calculated as follows: number of days/ week $\times$ number of hours/day $\times$ MET of a specific type of activity $=$ MET-h/week

The subjects completed an interviewer-administered FFQ that involved eighty-one food items and relevant dietary habits. The FFQ has been validated and tested for reproducibility ${ }^{(27)}$, and has been used in previous studies ${ }^{(28)}$. The food groups mainly included cereal products, soya and soya products, vegetables, fruit, red and processed meat, poultry, fish and other seafood, egg, dairy products and nuts. Dietary information was obtained from the cases during the preceding 12 months before diagnosis and from controls during the year before interview. The subjects were asked to report how often they consumed each food and how much they ate each time. Food photographs were used to help participants to quantify their dietary intakes.

The average daily intakes of total carbohydrate, fibre and starch were measured by summing the product of the frequency of consumption, portion consumed each time and the nutrient contents of each food item. The 2002 Chinese Food Composition Table was used to calculate energy, carbohydrate, fibre and other nutrient intakes ${ }^{(29)}$, and starch value was obtained from the US Department of Agriculture Food Composition Database ${ }^{(30)}$. The intake of non-fibre carbohydrate was calculated by excluding the total fibre intake from the total carbohydrate. The GI values of major carbohydrate-contributing foods (93.03\% of total carbohydrate) were obtained from the Chinese Food Composition Table ${ }^{(29)}$ and supplemented by the online GI database from University of Sydney (www.glycemicindex.com). The values of GI from Chinese Food Composition Table used glucose as the reference food. GL represents the glycaemic effect of food, and this effect is inherently a function of the carbohydrate available for digestion and absorption ${ }^{(31)}$. To calculate the GL, the available carbohydrate content was defined as the non-fibre carbohydrate, which excluded fibre from the total carbohydrate. Thus, the GL of a food was calculated by multiplying the non-fibre carbohydrate content of the food by its GI value, and then dividing the total by 100 . Dietary GL for a participant was the sum of the GL of all food items ${ }^{(13)}$. Overall dietary GI for each participant was derived from the sum of the GI of each food consumed, multiplied by the average daily amount consumed and non-fibre carbohydrate content, all divided by the daily intake of the non-fibre carbohydrate $^{(13)}$.

\section{Statistical analysis}

The $t$ test or Wilcoxon signed-ranks test was used for the continuous variables, and the $\chi^{2}$-test was used for the categorical variables to test the difference between the cases and controls. Dietary nutrient intake, GI and GL was adjusted based on total energy intake of men and women, using the regression residual method $^{(32)}$. Quartiles (Q1-Q4) of dietary intakes of total carbohydrate, non-fibre carbohydrate, total fibre and starch, dietary GI and GL were defined based on the distribution among the controls. Unconditional logistic regression model was used to estimate the OR and 95\% CI for the associations between dietary intakes of total carbohydrate, non-fibre carbohydrate, total fibre and starch, dietary GI and GL and colorectal cancer risk. The lowest-quartile group (Q1) served as the reference group. According to the characteristics comparison between cases and controls, or previous reported confounders, the following variables were selected in the multivariable-adjusted models: age, sex, marital status, residence, education, occupation, income level, BMI, active smoking, passive smoking, alcohol drinking, family history of cancer, occupational physical activity, household and leisure-time activities. All confounding factors were included as categorical variables except for age, BMI, household and leisure activities, which were regarded as continuous variables. Tests for trend were performed by entering the categorical variables (Q1-Q4) as continuous variables in the regression models; the quartiles (Q1-Q4) were coded as 1, 2, 3 and 4, respectively. Stratified analysis by sex, subgroup analysis by cancer site (colon or rectal cancer) and by sources of controls (community-derived controls and hospital-derived controls) were conducted. Sensitivity analysis was also performed by excluding study subjects who had physician-diagnosed diabetes mellitus. All of the statistical analyses were performed using SPSS 20.0 and the significant level was set at 0.05 (two-sided).

\section{Results}

There were 1944 cases in total, and 1079 of them were men and 865 were women. Among the cases, 1172 colon cancers and 772 rectal cancers were diagnosed. Table 1 shows the distribution of participants' socio-demographic and relevant characteristics. Compared with control subjects, more cases were married and lived in rural area, were less educated, had a higher proportion of farmers and lower income, tended to have more active smoking and passive smoking, had a higher frequency of alcohol drinking, reported having a first-degree relative with cancer, engaged in more occupational activities 
Table 1. Demographic and selected risk factors of colorectal cancer cases and controls in Chinese population*

(Numbers and percentages; mean values and standard deviations; medians and 25th, 75th percentiles)

\begin{tabular}{|c|c|c|c|c|c|}
\hline & \multicolumn{2}{|c|}{$\begin{array}{l}\text { Cases } \\
(n \text { 1944) }\end{array}$} & \multicolumn{2}{|c|}{$\begin{array}{l}\text { Controls } \\
(n \text { 2027) }\end{array}$} & \multirow[b]{2}{*}{$P$} \\
\hline & $n$ & $\%$ & $n$ & $\%$ & \\
\hline Age (years) & \multirow{2}{*}{\multicolumn{2}{|c|}{$56 \cdot 4$}} & \multirow{2}{*}{\multicolumn{2}{|c|}{$56 \cdot 2$}} & 0.50 \\
\hline Mean & & & & & \\
\hline SD & \multicolumn{2}{|c|}{$10 \cdot 0$} & \multicolumn{2}{|c|}{9.7} & \\
\hline \multicolumn{5}{|l|}{ Sex } & $0 \cdot 10$ \\
\hline Men & 1079 & $55 \cdot 5$ & 1073 & 52.9 & \\
\hline Women & 865 & 44.5 & 954 & $47 \cdot 1$ & \\
\hline \multicolumn{5}{|l|}{ Marital status } & 0.02 \\
\hline Married & 1844 & 94.9 & 1887 & $93 \cdot 1$ & \\
\hline Unmarried/divorces/widowed & 100 & $5 \cdot 1$ & 140 & $6 \cdot 9$ & \\
\hline \multicolumn{5}{|l|}{ Residence } & $<0.01$ \\
\hline Urban & 1241 & 63.8 & 1604 & $79 \cdot 2$ & \\
\hline Rural & 703 & $36 \cdot 2$ & 422 & 20.8 & \\
\hline \multicolumn{5}{|l|}{ Educational level } & $<0.01$ \\
\hline Primary school or below & 622 & $32 \cdot 0$ & 418 & $20 \cdot 6$ & \\
\hline Secondary school & 532 & $27 \cdot 4$ & 496 & 24.5 & \\
\hline High school & 467 & $24 \cdot 0$ & 573 & $28 \cdot 3$ & \\
\hline College or above & 323 & $16 \cdot 6$ & 540 & $26 \cdot 6$ & \\
\hline \multicolumn{5}{|l|}{ Occupation } & $<0.01$ \\
\hline $\begin{array}{l}\text { Administrator/other white } \\
\text { collar worker }\end{array}$ & 259 & $13 \cdot 3$ & 350 & $17 \cdot 3$ & \\
\hline Blue collar worker & 405 & $20 \cdot 8$ & 415 & 20.5 & \\
\hline Farmer/other & 1280 & $65 \cdot 8$ & 1259 & $62 \cdot 2$ & \\
\hline \multicolumn{5}{|l|}{ Income (Yuan/month) } & $<0.01$ \\
\hline$<2000$ & 288 & $14 \cdot 8$ & 227 & $11 \cdot 2$ & \\
\hline $2001-5000$ & 593 & $30 \cdot 5$ & 687 & 34.0 & \\
\hline $5001-8000$ & 569 & $29 \cdot 3$ & 684 & 33.8 & \\
\hline$>8001$ & 494 & $25 \cdot 4$ & 423 & 20.9 & \\
\hline \multicolumn{5}{|l|}{$\mathrm{BMI}\left(\mathrm{kg} / \mathrm{m}^{2}\right)$} & 0.73 \\
\hline Mean & \multirow{2}{*}{\multicolumn{2}{|c|}{$\begin{array}{c}23 \cdot 2 \\
3 \cdot 3\end{array}$}} & \multirow{2}{*}{\multicolumn{2}{|c|}{$\begin{array}{c}23 \cdot 3 \\
3 \cdot 1\end{array}$}} & \\
\hline SD & & & & & \\
\hline Active smoking & 745 & $38 \cdot 3$ & 624 & $30 \cdot 8$ & $<0.01$ \\
\hline Passive smoking & 879 & $45 \cdot 2$ & 549 & $27 \cdot 1$ & $<0.01$ \\
\hline Regular drinker & 344 & $17 \cdot 7$ & 267 & $13 \cdot 2$ & $<0.01$ \\
\hline Family history of cancer & 268 & $13 \cdot 8$ & 169 & $8 \cdot 3$ & $<0.01$ \\
\hline \multicolumn{5}{|l|}{ Occupational activity } & $<0.01$ \\
\hline Non-working & 729 & 37.5 & 932 & $46 \cdot 0$ & \\
\hline Sedentary & 370 & $19 \cdot 0$ & 312 & 15.4 & \\
\hline Light occupation & 344 & $17 \cdot 7$ & 463 & $22 \cdot 8$ & \\
\hline Moderate occupation & 241 & $12 \cdot 4$ & 204 & $10 \cdot 1$ & \\
\hline Heavy-activity occupation & 260 & 13.4 & 116 & $5 \cdot 7$ & \\
\hline \multicolumn{5}{|l|}{$\begin{array}{l}\text { Household and leisure-time } \\
\text { activities (MET-h/week) }\end{array}$} & $<0.01$ \\
\hline Median & \multirow{2}{*}{\multicolumn{2}{|c|}{$\begin{array}{c}28 \cdot 9 \\
8 \cdot 8,52 \cdot 5\end{array}$}} & \multicolumn{2}{|c|}{35.4} & \\
\hline 25th, 75th percentiles & & & \multicolumn{2}{|c|}{$17 \cdot 0,55 \cdot 9$} & \\
\hline $\begin{array}{l}\text { Age at menarche (years) } \dagger \\
\text { Mean } \\
\text { SD }\end{array}$ & $14 \cdot 7$ & $2 \cdot 5$ & $14 \cdot 6$ & $2 \cdot 3$ & 0.44 \\
\hline Menopausal status $\dagger$ & & & & & 0.25 \\
\hline Premenopausal & 248 & $28 \cdot 7$ & 297 & $31 \cdot 1$ & \\
\hline Postmenopausal & 617 & 71.3 & 657 & 68.9 & \\
\hline
\end{tabular}

MET, metabolic equivalent task.

* Continuous variables were evaluated using $t$ tests or Wilcoxon rank-sum tests. Categorical variables were evaluated using $x^{2}$ tests.

$\dagger$ Among the subgroup of women.

and engaged in fewer household and leisure activities. No statistically significant differences were found in terms of age, sex, BMI, women's postmenopausal status and age at menarche between cases and controls.

Compared with the controls, the cases had a higher dietary GI and GL, a higher intake of starch and a lower intake of total energy and total fibre. Total carbohydrate intake was 265.0 (SD 50.2) $\mathrm{g} / \mathrm{d}$ for cases and 267.8 (SD 50.5 ) g/d for controls; the intake of non-fibre carbohydrate was 255.8 (SD 49.7) $\mathrm{g} / \mathrm{d}$ for cases and 257.3 (SD 49.9) g/d for controls. Nevertheless, neither total carbohydrate nor non-fibre carbohydrate was observed to be statistically significantly different between two groups (Table 2).

Among control subjects, two main food sources of total carbohydrate, non-fibre carbohydrate, fibre, starch and GL were cereal grains, vegetable and fruit. Cereal grains contributed $75.34 \%$ of total carbohydrate, $77.67 \%$ of non-fibre carbohydrate, $91.29 \%$ of starch and $87.95 \%$ of GL. Vegetables and fruit contributed $18.08 \%$ of total carbohydrate, $16.07 \%$ of nonfibre carbohydrate, $6.34 \%$ of starch and $9.82 \%$ of GL. However, vegetables and fruit contributed $61.31 \%$ and cereal grains contributed $25.58 \%$ of total fibre. As for daily GI, cereal grains, vegetables and fruit contributed 69.15 and $21.49 \%$ to non-fibre carbohydrate for the first quartile of GI, and 84.73 and $11.23 \%$ to non-fibre carbohydrate for the last quartile of GI.

The OR and $95 \% \mathrm{CI}$ of colorectal cancer for the intakes of total carbohydrate, non-fibre carbohydrate, total fibre, starch, dietary GI and GL are presented in Table 3. Total carbohydrate intake was not found to be associated with colorectal cancer risk, with an adjusted OR of 0.85 (95\% CI 0.70, 1.03) comparing the highest with the lowest quartile $\left(P_{\text {trend }}=0.08\right)$. For the intake of total fibre, the highest quartile intake showed a risk reduction of $53 \%$ compared with the lowest quartile (OR 0.47; $95 \%$ CI $\left.0.39,0.58, P_{\text {trend }}<0.01\right)$. Dietary GI was positively associated with colorectal cancer risk, with an adjusted OR of 3.10 (95\% CI $2.51,3.85, P_{\text {trend }}<0.01$ ) comparing the highest with the lowest quartile. However, no significant association was found between the intakes of non-fibre carbohydrate, starch, dietary GL and colorectal cancer risk.

Stratified analysis by sex showed that dietary GI was positively associated with colorectal cancer among both sexes. The adjusted OR for the highest quartile $v$. the lowest quartile was $3.49\left(95 \%\right.$ CI 2.62, 4.64, $\left.P_{\text {trend }}<0.01\right)$ for men and 2.44 (95\% CI $\left.1.72,3.46, P_{\text {trend }}<0.01\right)$ for women. Intakes of total carbohydrate, non-fibre carbohydrate and total fibre were inversely associated with colorectal cancer risk among men but not among women. Compared with the lowest quartile, the adjusted OR of the highest quartile was 0.66 (95\% CI 0.51, 0.85, $\left.P_{\text {trend }}<0.01\right)$ for total carbohydrate, 0.72 (95\% CI $0.55,0.93$, $\left.P_{\text {trend }}=0.01\right)$ for non-fibre carbohydrate and 0.36 (95\% CI 0.27 , $\left.0 \cdot 47, P_{\text {trend }}<0 \cdot 01\right)$ for total fibre among men. Among women, dietary GL was positively related to colorectal cancer risk. The adjusted OR of the highest quartile compared with the lowestquartile intake was 1.42 (95\% CI 1.04, 1.95, $\left.P_{\text {trend }}<0.01\right)$. No significant association was found between consumption of total carbohydrate, non-fibre carbohydrate or total fibre and colorectal cancer risk among women. In addition, there was no notable association between the intake of starch and colorectal cancer risk in either sex (Table 4).

As presented in Table 5, subgroup analysis by cancer site was similar to the analysis among all colorectal cancer cases. Subgroup analysis by community-derived controls and hospitalderived controls also showed that no significant difference between the intakes of non-fibre carbohydrate, fibre, starch, dietary GI and GL and colorectal cancer risk was found when 
Table 2. Intakes of energy, total carbohydrate, non-fibre carbohydrate, total fibre, starch, glycaemic index and glycaemic load among case and control subjects in Guangdong, China*

(Mean values and standard deviations; medians and 25th, 75th percentiles)

\begin{tabular}{|c|c|c|c|c|c|c|c|c|c|}
\hline & \multicolumn{4}{|c|}{ Cases $(n 1944)$} & \multicolumn{4}{|c|}{ Controls (n 2027) } & \multirow[b]{2}{*}{$P$} \\
\hline & Mean & SD & Median & 25th, 75 th percentiles & Mean & SD & Median & 25th, 75 th percentiles & \\
\hline Energy (kJ/d) & 7201 & 2133.0 & 6954 & 5661,8435 & 7628 & $2360 \cdot 2$ & 7263 & 5966,8895 & $<0.01$ \\
\hline Energy (kcal/d) & 1721 & 509.8 & 1662 & 1353,2016 & 1823 & $564 \cdot 1$ & 1736 & 1426,2126 & $<0.01$ \\
\hline Total carbohydrate $(\mathrm{g} / \mathrm{d}) \dagger$ & $265 \cdot 0$ & $50 \cdot 2$ & $262 \cdot 6$ & $230 \cdot 7,300 \cdot 2$ & 267.8 & $50 \cdot 5$ & 262.5 & $230 \cdot 1,305 \cdot 8$ & 0.26 \\
\hline Non-fibre carbohydrate $(g / d) \dagger$ & $255 \cdot 8$ & $49 \cdot 7$ & 253.5 & $221 \cdot 8,290 \cdot 6$ & $257 \cdot 3$ & 49.9 & $251 \cdot 7$ & $220 \cdot 0,294 \cdot 7$ & 0.74 \\
\hline Total fibre $(g / d) \dagger$ & $10 \cdot 6$ & $3 \cdot 3$ & $10 \cdot 2$ & $8 \cdot 3,12 \cdot 3$ & 11.7 & 3.3 & 11.4 & $9.4,13.6$ & $<0.01$ \\
\hline Starch $(g / d) \dagger$ & $98 \cdot 1$ & $23 \cdot 0$ & 97.1 & $82 \cdot 2,114 \cdot 0$ & $96 \cdot 7$ & $23 \cdot 1$ & 94.5 & $80 \cdot 1,112 \cdot 3$ & 0.01 \\
\hline Glycaemic index $†$ & $68 \cdot 7$ & 4.0 & 69.1 & $66 \cdot 5,71 \cdot 4$ & $66 \cdot 8$ & $4 \cdot 3$ & $67 \cdot 3$ & $64 \cdot 3,69 \cdot 8$ & $<0.01$ \\
\hline Glycaemic load $\dagger$ & $165 \cdot 7$ & 38.9 & $162 \cdot 3$ & $139.4,193.0$ & $162 \cdot 2$ & $38 \cdot 3$ & $157 \cdot 7$ & $134 \cdot 4,187 \cdot 2$ & $<0.01$ \\
\hline
\end{tabular}

* Wilcoxon rank-sum test comparing the median consumption levels between cases and controls.

$\dagger$ Adjusted for the consumption for total energy intake by the regression residual method.

Table 3. Colorectal cancer according to quartiles (Q) of the intakes of total carbohydrate, non-fibre carbohydrate, total fibre, starch, dietary glycaemic index and glycaemic load

(Odds ratios and $95 \%$ confidence intervals)

\begin{tabular}{|c|c|c|c|c|c|c|c|c|}
\hline & \multirow[b]{2}{*}{ Q1 } & \multicolumn{2}{|c|}{ Q2 } & \multicolumn{2}{|c|}{ Q3 } & \multicolumn{2}{|c|}{ Q4 } & \multirow[b]{2}{*}{$P$} \\
\hline & & OR & $95 \% \mathrm{Cl}$ & OR & $95 \% \mathrm{Cl}$ & OR & $95 \% \mathrm{Cl}$ & \\
\hline \multicolumn{9}{|l|}{ Total carbohydrate } \\
\hline Median intake $(\mathrm{g} / \mathrm{d})$ & $212 \cdot 0$ & \multirow{2}{*}{\multicolumn{2}{|c|}{$\begin{array}{c}274.5 \\
437 / 508\end{array}$}} & \multicolumn{2}{|c|}{304.1} & \multicolumn{2}{|c|}{$330 \cdot 7$} & \\
\hline No. cases/controls & $586 / 506$ & & & \multicolumn{2}{|c|}{$423 / 507$} & \multicolumn{2}{|c|}{$498 / 506$} & \\
\hline Crude & 1 & 0.74 & $0.62,0.89$ & 0.72 & $0.60,0.86$ & 0.85 & $0.72,1.01$ & 0.05 \\
\hline Adjusted* & 1 & 0.75 & $0.62,0.90$ & 0.74 & $0.61,0.90$ & 0.85 & $0.70,1.03$ & 0.08 \\
\hline \multicolumn{9}{|l|}{ Non-fibre carbohydrate } \\
\hline Median intake $(\mathrm{g} / \mathrm{d})$ & 202.5 & \multirow{2}{*}{\multicolumn{2}{|c|}{$\begin{array}{c}262.5 \\
449 / 507\end{array}$}} & \multicolumn{2}{|c|}{$292 \cdot 4$} & \multicolumn{2}{|c|}{319.8} & \\
\hline No. cases/controls & $554 / 506$ & & & & 508 & & 506 & \\
\hline Crude & 1 & 0.81 & $0.68,0.96$ & 0.77 & $0.65,0.92$ & 0.92 & $0.78,1 \cdot 10$ & 0.30 \\
\hline Adjusted* & 1 & 0.79 & $0.65,0.95$ & 0.78 & $0.65,0.95$ & 0.90 & $0.74,1.09$ & 0.27 \\
\hline \multicolumn{9}{|l|}{ Total fibre } \\
\hline Median intake $(\mathrm{g} / \mathrm{d})$ & $8 \cdot 3$ & \multirow{2}{*}{\multicolumn{2}{|c|}{$\begin{array}{c}10 \cdot 4 \\
491 / 508\end{array}$}} & \multicolumn{2}{|c|}{$12 \cdot 4$} & \multicolumn{2}{|c|}{$15 \cdot 5$} & \\
\hline No. cases/controls & $779 / 506$ & & & & 507 & & 506 & \\
\hline Crude & 1 & 0.63 & $0.53,0.74$ & 0.46 & $0.38,0.55$ & 0.41 & $0.34,0.49$ & $<0.01$ \\
\hline Adjusted* & 1 & 0.65 & $0.55,0.79$ & 0.50 & $0.42,0.61$ & 0.47 & $0.39,0.58$ & $<0.01$ \\
\hline \multicolumn{9}{|l|}{ Starch } \\
\hline Median intake $(\mathrm{g} / \mathrm{d})$ & $72 \cdot 0$ & \multicolumn{2}{|c|}{$95 \cdot 7$} & \multicolumn{2}{|c|}{$110 \cdot 7$} & \multicolumn{2}{|c|}{$125 \cdot 6$} & \\
\hline No. cases/controls & $483 / 506$ & \multicolumn{2}{|c|}{$427 / 507$} & \multicolumn{2}{|c|}{$504 / 508$} & \multicolumn{2}{|c|}{$530 / 506$} & \\
\hline Crude & 1 & 0.88 & $0.74,1.06$ & 1.04 & $0.87,1.24$ & $1 \cdot 10$ & $0.92,1.31$ & 0.12 \\
\hline Adjusted* & 1 & 0.90 & $0.74,1.09$ & 1.07 & $0.88,1.30$ & 1.04 & $0.85,1.26$ & 0.37 \\
\hline \multicolumn{9}{|l|}{ Glycaemic index } \\
\hline Median value & $61 \cdot 8$ & \multirow{2}{*}{\multicolumn{2}{|c|}{$\begin{array}{c}65 \cdot 9 \\
380 / 507\end{array}$}} & \multicolumn{2}{|c|}{68.4} & & & \\
\hline No. cases/controls & $226 / 506$ & & & & 508 & & 506 & \\
\hline Crude & 1 & 1.68 & $1.37,2.06$ & $2 \cdot 26$ & $1 \cdot 85,2 \cdot 75$ & 3.66 & $3.02,4.43$ & $<0.01$ \\
\hline Adjusted* & 1 & 1.73 & $1 \cdot 38,2 \cdot 16$ & $2 \cdot 10$ & $1 \cdot 69,2.61$ & 3.10 & $2.51,3.85$ & $<0.01$ \\
\hline Glycaemic load & & & & & & & & \\
\hline Median value & 121.4 & & & & & & & \\
\hline No. cases/controls & $485 / 506$ & & 508 & & 507 & & 506 & \\
\hline Crude & 1 & 0.81 & $0.67,0.97$ & 0.97 & $0.81,1.15$ & 1.23 & $1.04,1.46$ & $<0.01$ \\
\hline Adjusted* & 1 & 0.77 & $0.63,0.94$ & 0.96 & $0.79,1.16$ & 1.14 & $0.94,1.39$ & 0.05 \\
\hline
\end{tabular}

* OR was adjusted for age, sex, marital status, residence, education, occupation, income level, smoking status, passive smoking, alcohol drinking, family history of cancer, occupational physical activity, household and leisure-time activities.

using either group, except that total carbohydrate intake was inversely associated with colorectal cancer risk when using the community-derived controls (Table 6).

In the present study, there were 159 colorectal cancer cases (8.18\%) and 113 controls $(5 \cdot 57 \%)$ with physician-diagnosed diabetes mellitus. The prevalence of physician-diagnosed diabetes mellitus was significantly higher in colorectal cancer cases than in controls. Sensitivity analysis excluding study subjects with diabetes mellitus did not materially change the results (data not shown).

\section{Discussion}

This case-control study found that dietary GI was positively associated with colorectal cancer risk, whereas total fibre intake was negatively associated with colorectal cancer risk. However, 
Table 4. Colorectal cancer according to quartiles (Q) of the intakes of total carbohydrate, non-fibre carbohydrate, total fibre, starch, dietary glycaemic index and glycaemic load by sex (Odds ratios and $95 \%$ confidence intervals)

\begin{tabular}{|c|c|c|c|c|c|c|c|c|c|c|c|c|c|c|c|c|c|}
\hline & \multicolumn{8}{|c|}{ Men ( $n$ 1079) } & \multicolumn{8}{|c|}{ Women (n 865) } & \multirow[b]{3}{*}{$P_{\text {interaction }}$} \\
\hline & \multirow[b]{2}{*}{ Q1 } & \multicolumn{2}{|r|}{ Q2 } & \multicolumn{2}{|r|}{ Q3 } & \multicolumn{2}{|r|}{ Q4 } & \multirow[b]{2}{*}{$P_{\text {trend }}$} & \multirow[b]{2}{*}{ Q1 } & \multicolumn{2}{|r|}{ Q2 } & \multicolumn{2}{|r|}{ Q3 } & \multicolumn{2}{|r|}{ Q4 } & \multirow[b]{2}{*}{$P_{\text {trend }}$} & \\
\hline & & OR & $95 \% \mathrm{Cl}$ & OR & $95 \% \mathrm{Cl}$ & OR & $95 \% \mathrm{Cl}$ & & & OR & $95 \% \mathrm{Cl}$ & OR & $95 \% \mathrm{Cl}$ & OR & $95 \% \mathrm{Cl}$ & & \\
\hline Total carbohydrate & & & & & & & & & & & & & & & & & \multirow[t]{5}{*}{$<0.01$} \\
\hline Median intake $(\mathrm{g} / \mathrm{d})$ & 251.4 & \multirow{2}{*}{\multicolumn{2}{|c|}{$\begin{array}{c}288.5 \\
264 / 269\end{array}$}} & \multirow{2}{*}{\multicolumn{2}{|c|}{$\begin{array}{c}315.9 \\
220 / 268\end{array}$}} & \multirow{2}{*}{\multicolumn{2}{|c|}{$\begin{array}{c}348 \cdot 4 \\
214 / 268\end{array}$}} & & $200 \cdot 0$ & \multirow{2}{*}{\multicolumn{2}{|c|}{$\begin{array}{c}223.5 \\
173 / 239\end{array}$}} & \multirow{2}{*}{\multicolumn{2}{|c|}{$\begin{array}{c}242.4 \\
203 / 239\end{array}$}} & \multirow{2}{*}{\multicolumn{2}{|c|}{$\begin{array}{c}264.8 \\
284 / 238\end{array}$}} & \multirow[b]{3}{*}{$<0.01$} & \\
\hline No. cases/controls & $381 / 268$ & & & & & & & & $205 / 238$ & & & & & & & & \\
\hline Crude & 1 & 0.69 & $0.55,0.87$ & 0.58 & $0.46,0.73$ & 0.56 & $0.44,0.71$ & $<0.01$ & 1 & 0.84 & $0.64,1.10$ & 0.99 & $0.76,1.28$ & 1.39 & $1.08,1.79$ & & \\
\hline Adjusted ${ }^{*}$ & 1 & 0.72 & $0.56,0.93$ & 0.63 & $0.49,0.82$ & 0.66 & $0.51,0.85$ & $<0.01$ & 1 & 0.83 & $0.61,1.14$ & 0.95 & $0.69,1.30$ & 1.24 & $0.91,1.68$ & 0.12 & \\
\hline Non-fibre carbohydrate & & & & & & & & & & & & & & & & & $<0.01$ \\
\hline Median intake $(\mathrm{g} / \mathrm{d})$ & $240 \cdot 7$ & & $277 \cdot 4$ & & 304.4 & & 336.7 & & $190 \cdot 4$ & & 214.5 & & $232 \cdot 2$ & & 254.0 & & \\
\hline No. cases/controls & $352 / 268$ & & $82 / 268$ & & $23 / 269$ & & $22 / 268$ & & $202 / 238$ & & $67 / 239$ & & 206/239 & & $90 / 238$ & & \\
\hline Crude & 1 & 0.80 & $0.64,1.01$ & 0.63 & $0.50,0.80$ & 0.63 & $0.50,0.80$ & $<0.01$ & 1 & 0.82 & $0.63,1.08$ & 1.02 & $0.78,1.32$ & 1.44 & $1.11,1.85$ & $<0.01$ & \\
\hline Adjusted* & 1 & 0.78 & $0.61,0.99$ & 0.69 & $0.53,0.89$ & 0.72 & $0.55,0.93$ & 0.01 & 1 & 0.85 & $0.62,1.16$ & 0.96 & $0.70,1.31$ & 1.27 & $0.93,1.73$ & 0.09 & \\
\hline Total fibre & & & & & & & & & & & & & & & & & $<0.01$ \\
\hline Median intake $(\mathrm{g} / \mathrm{d})$ & 8.9 & & $11 \cdot 0$ & & $13 \cdot 0$ & & $16 \cdot 3$ & & 7.8 & & 9.7 & & 11.5 & & $14 \cdot 3$ & & \\
\hline No. cases/controls & $473 / 268$ & & $73 / 269$ & & $71 / 268$ & & $62 / 268$ & & $306 / 238$ & & $18 / 239$ & & $86 / 239$ & & $55 / 238$ & & \\
\hline Crude & 1 & 0.58 & $0.46,0.72$ & 0.36 & $0.28,0.46$ & 0.34 & $0.27,0.44$ & $<0.01$ & 1 & 0.71 & $0.55,0.91$ & 0.61 & $0.47,0.78$ & 0.51 & $0.39,0.66$ & $<0.01$ & \\
\hline Adjusted* & 1 & 0.59 & $0.46,0.75$ & 0.35 & $0.27,0.46$ & 0.36 & $0.27,0.47$ & $<0.01$ & 1 & 0.80 & $0.60,1.08$ & 0.81 & $0.59,1.09$ & 0.76 & $0.55,1.05$ & 0.10 & \\
\hline Starch & & & & & & & & & & & & & & & & & $<0.01$ \\
\hline Median intake $(\mathrm{g} / \mathrm{d})$ & 85.1 & & $102 \cdot 0$ & & $116 \cdot 5$ & & 133.4 & & 65.5 & & 77.8 & & 86.9 & & 99.6 & & \\
\hline No. cases/controls & $296 / 268$ & & $76 / 268$ & & $72 / 269$ & & $35 / 268$ & & $187 / 238$ & & $51 / 239$ & & $32 / 239$ & & $95 / 238$ & & \\
\hline Crude & 1 & 0.93 & $0.74,1.18$ & 0.92 & $0.72,1.16$ & 0.79 & $0.62,1.01$ & 0.07 & 1 & 0.80 & $0.61,1.06$ & 1.24 & $0.95,1.61$ & 1.58 & $1.22,2.04$ & $<0.01$ & \\
\hline Adjusted* & 1 & 0.97 & $0.75,1.25$ & 1.04 & $0.81,1.34$ & 0.92 & $0.70,1.19$ & 0.66 & 1 & 0.85 & $0.61,1.18$ & 1.20 & $0.88,1.63$ & 1.21 & $0.88,1.65$ & 0.08 & \\
\hline Glycaemic index & & & & & & & & & & & & & & & & & 0.14 \\
\hline Median value & $62 \cdot 8$ & & 66.5 & & 69.0 & 71.7 & & & $60 \cdot 8$ & & $65 \cdot 2$ & & 67.9 & & 71.1 & & \\
\hline No. cases/controls & $127 / 268$ & & $98 / 268$ & & $92 / 269$ & $462 / 2$ & & & $99 / 238$ & & $82 / 239$ & & $20 / 239$ & & $64 / 238$ & & \\
\hline Crude & 1 & 1.56 & $1 \cdot 18,2 \cdot 06$ & 2.29 & $1.75,3.00$ & 3.64 & $2.81,4.72$ & $<0.01$ & 1 & 1.83 & $1.35,2.48$ & $2 \cdot 21$ & $1.64,2.98$ & 3.68 & $2.76,4.89$ & $<0.01$ & \\
\hline Adjusted* ${ }^{*}$ & 1 & 1.60 & $1 \cdot 18,2 \cdot 16$ & 2.41 & $1.80,3.22$ & 3.49 & $2 \cdot 62,4.64$ & $<0.01$ & 1 & 2.08 & $1.46,2.97$ & 1.77 & $1.25,2.52$ & 2.44 & $1.72,3.46$ & $<0.01$ & \\
\hline Glycaemic load & & & & & & & & & & & & & & & & & $<0.01$ \\
\hline Median value & $146 \cdot 0$ & & $172 \cdot 1$ & & $194 \cdot 3$ & & $224 \cdot 6$ & & 111.1 & & $130 \cdot 9$ & & 143.9 & & $164 \cdot 8$ & & \\
\hline No. cases/controls & $311 / 268$ & & $42 / 269$ & & $56 / 268$ & & $70 / 268$ & & $174 / 238$ & & $50 / 239$ & & $13 / 239$ & & $28 / 238$ & & \\
\hline Crude & 1 & 0.78 & $0.61,0.98$ & 0.82 & $0.65,1.04$ & 0.87 & $0.69,1 \cdot 10$ & 0.31 & 1 & 0.86 & $0.65,1.14$ & 1.22 & $0.93,1.60$ & 1.89 & $1.46,2.44$ & $<0.01$ & \\
\hline Adjusted* & 1 & 0.82 & $0.64,1.07$ & 0.91 & $0.70,1.18$ & 0.99 & $0.76,1.28$ & 0.94 & 1 & 0.72 & $0.51,1.00$ & 1.06 & $0.77,1.46$ & 1.42 & $1.04,1.95$ & $<0.01$ & \\
\hline
\end{tabular}

* OR was adjusted for age, marital status, residence, education, occupation, income level, smoking status, passive smoking, alcohol drinking, family history of cancer, occupational physical activity, household and leisure-time activities. 
Table 5. Colorectal cancer according to quartiles $(\mathrm{Q})$ of the intakes of total carbohydrate, non-fibre carbohydrate, total fibre, starch, dietary glycaemic index and glycaemic load by cancer site (Odds ratios and $95 \%$ confidence intervals)

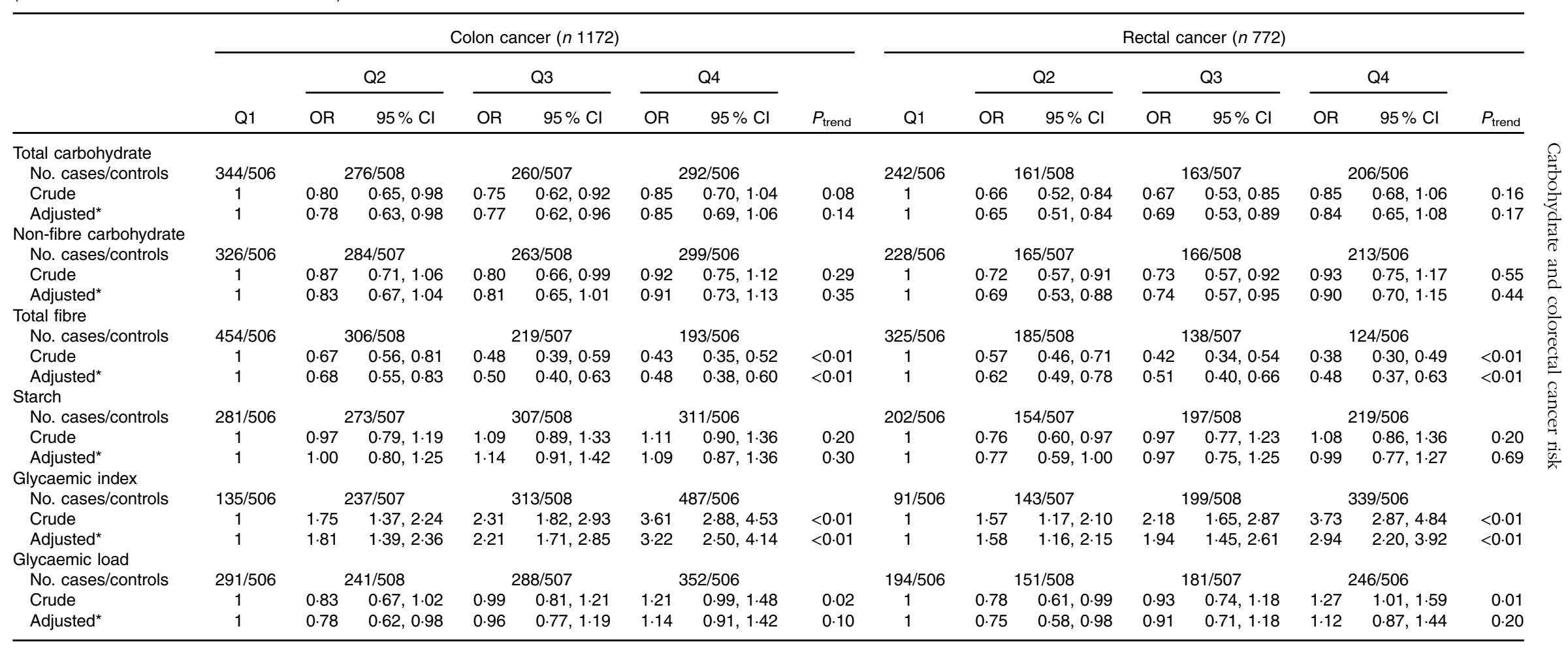


Table 6. Colorectal cancer by quartiles $(Q)$ of total carbohydrate, non-fibre carbohydrate, total fibre, starch, dietary glycaemic index and glycaemic load in two control groups (Odds ratios and $95 \%$ confidence intervals)

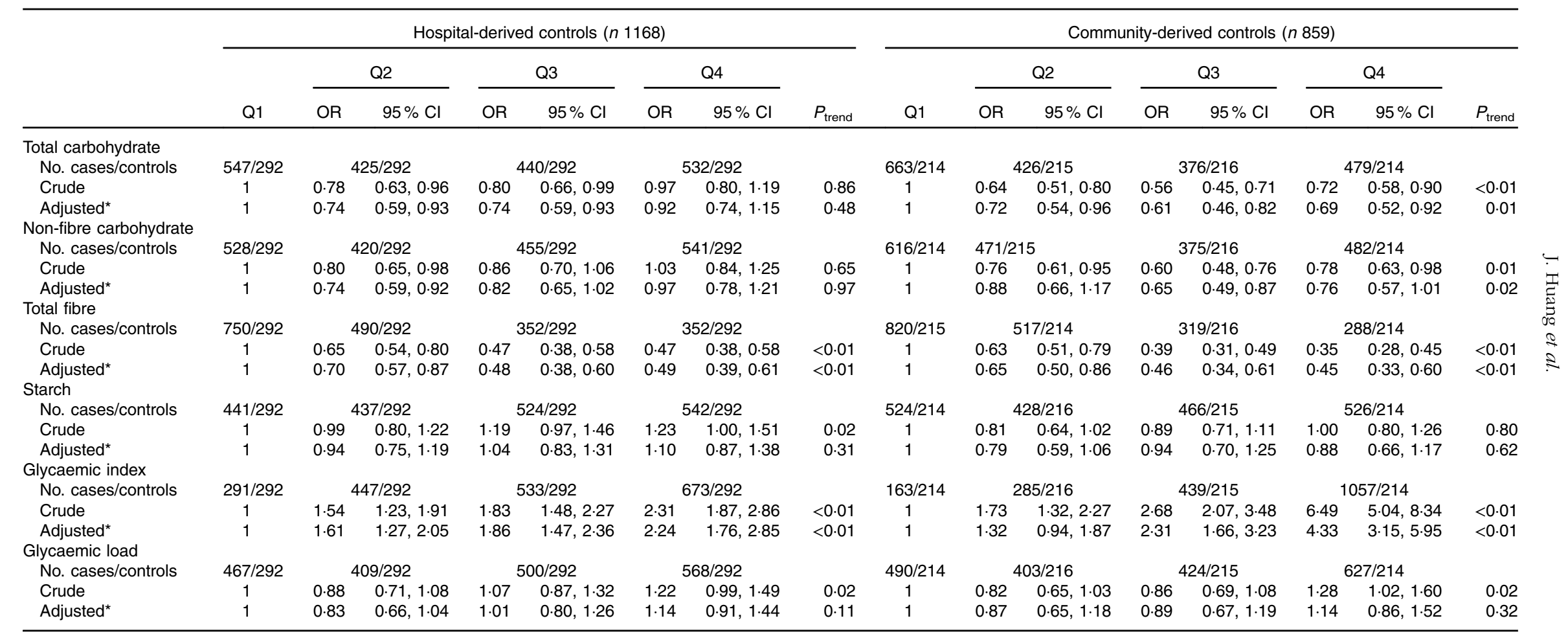

* OR was adjusted for age, sex, marital status, residence, education, occupation, income level, smoking status, passive smoking, alcohol drinking, family history of cancer, occupational physical activity, household and leisure-time activities. 
there was no clear association between total carbohydrate, non-fibre carbohydrate, starch or dietary GL and colorectal cancer risk.

In 2012 , three meta-analyses ${ }^{(11,15,16)}$ were conducted to detect the association between dietary carbohydrate, GI and GL and colorectal cancer risk. Our findings were partially consistent with them. Regarding dietary carbohydrate, a metaanalysis including fourteen cohort studies concluded that there was no clear association between dietary total carbohydrate and colorectal cancer; the summary RR for high $v$. low intake was $1.00(95 \% \text { CI } 0 \cdot 87,1 \cdot 14)^{(11)}$. For dietary GI, two meta-analyses including the same twelve cohort studies observed a borderline positive association between dietary GI and colorectal cancer risk; the summary RR for high $v$. low intake was 1.07 (95\% CI $0.99,1.16)^{(11)}$ and $1.08(95 \% \text { CI } 1.00,1.17)^{(16)}$, respectively. Similarly, a meta-analysis analysing eight cohort studies and five case-control studies also found that dietary GI was related to an increased risk of colon cancer; the RR for the highest intake was 1.17 (95\% CI 1.00, 1.36) compared with the lowest intake ${ }^{(15)}$. Furthermore, a cohort study conducted in Italy in 2015 with 47749 participants including 421 colorectal cancer cases ${ }^{(14)}$ found that high dietary GI and high carbohydrate intake from high-GI foods were associated with increased risk of colorectal cancer. Compared with the lowest quintile, the hazard ratios (HR) of the highest quintile were 1.35 (95\% CI 1.03, 1.78) and 1.45 (95\% CI 1.04, 2.03), respectively, whereas low-GI carbohydrate was in relation to a decreased risk of colorectal cancer (HR $0.73 ; 95 \%$ CI $0.54,0.98$, the highest quintile $v$. the lowest quintile). As regards dietary GL, the same cohort study mentioned above found that high dietary GL was significantly associated with increased risk of colon cancer ${ }^{(33)}$. However, a meta-analysis reported no significant association between dietary GL and colon cancer risk ${ }^{(15)}$, and another two metaanalyses also revealed a null association between dietary GL and colorectal cancer risk $^{(11,16)}$, which were consistent with our finding.

Fibre was the indigestible portion of total carbohydrate. Previous studies revealed that dietary fibre was probably a protective factor against colorectal cancer ${ }^{(17)}$. Therefore, the association between total carbohydrate and colorectal cancer risk might be biased by the protective effect of fibre. Some studies evaluated the association between dietary intake of nonfibre carbohydrate and colorectal cancer risk. The cohort study mentioned above found that carbohydrate intake excluding dietary fibre had no significant association with colorectal cancer risk $^{(14)}$, which was similar to our finding. However, a cohort study conducted in the USA with 38451 women including 174 colorectal cancer cases reported a positive association between non-fibre carbohydrate intake and colorectal cancer risk ${ }^{(18)}$. Another case-control study also drew the same conclusion ${ }^{(19)}$. One possible explanation for the inconsistent results might be that different studies adjusted for different confounders. In these two studies with positive results ${ }^{(18,19)}$, the important confounding factors such as physical activity, smoking status and alcohol use were not included in the multivariable regression models, which would bias the risk estimates and lead to a different conclusion from our study. In addition, the cohort study mentioned above ${ }^{(18)}$ only focused on women, which might potentially affect the generalisability of the conclusion.

Contrary to our initial assumption, the present study found no significant association between total carbohydrate intake and colorectal cancer risk. Lack of an association between total carbohydrate and colorectal cancer risk might partly attribute to dietary fibre and resistant starch. Fibre and resistant starch can be fermented by intestine flora, and then produced SCFA, such as butyrate ${ }^{(34)}$. Butyrate was important for the intestinal homoeostasis, such as modulating $\mathrm{PH}$ and epithelial thickness, decreasing inflammation ${ }^{(35)}$ and reducing expression of microRNA that are related to increase the proliferation colon cancer cell ${ }^{(36)}$. The protective effect of dietary fibre observed in the present study and the underlying resistant starch may have offset the adverse effect of other carbohydrate components, as it was presented that after excluding fibre from total carbohydrate, the OR of non-fibre carbohydrate was attenuated from 0.85 to 0.90 in our study.

In agreement with the findings from two previous metaanalyses ${ }^{(11,16)}$, the present study found no significant association between dietary GL and colorectal cancer risk. Cereal grains, vegetables and fruit contributed 87.95 and $9.82 \%$ to the daily dietary GL among control subjects. Although calculating GL excluded dietary fibre content, it was difficult to dissociate the effects of GL from other beneficial contents such as the phytochemicals in vegetables and fruit ${ }^{(37)}$. The protective effect of bioactive substances may dilute the adverse effect of GL. Therefore, it is possible that the relationship between dietary GL and colorectal cancer risk have been underestimated. More studies, especially prospective cohort studies, are required to clarify this issue.

To date, only two case-control studies examined the association between starch intake and colorectal cancer risk. One study from Italy including 1953 colorectal cancer cases and 4154 controls found a positive association between starch intake and colorectal cancer risk ${ }^{(20)}$. However, another study from Swiss, which recruited 286 colorectal cancer cases and 550 controls, did not observe a significant association ${ }^{(21)}$, which was consistent with our results. According to different digestive rate, starch was grouped into rapidly digestible starch, slowly digestible starch and resistant starch ${ }^{(38)}$. It was reported that resistant starch could escape digestion in the small intestine and was beneficial for bowel health, by increasing faecal $\mathrm{PH}$, faecal bulk, butyrate level and epithelial apoptosis and inhibiting cancer cell proliferation ${ }^{(39)}$. The absence of an association between starch intake and colorectal cancer risk found in the present study might partially be due to the existence of resistant starch, which is not digested in the human small intestine. Owing to the limited evidence on the relationship between starch intake and colorectal cancer risk, further studies are needed to verify this issue.

The present study showed that GI, but not total carbohydrate, non-fibre carbohydrate, starch or GL, was positively associated with colorectal cancer risk. This indicated that increased colorectal cancer risk may depend more on the quality of carbohydrate consumed rather than a carbohydrate-rich diet. Consistent with our findings, a cohort study ${ }^{(14)}$ reported that higher intake of carbohydrates from high-GI foods was 
positively associated with colorectal cancer risk (highest $v$. lowest quartile: adjusted HR 1.45; 95\% CI 1.04, 2.03, $P_{\text {trend }}=$ 0.03). However, higher intake of carbohydrates from low-GI foods was negatively associated with colorectal cancer risk (highest $v$. lowest quartile: adjusted HR 0.73; 95\% CI 0.54, 0.98, $P_{\text {trend }}=0.03$ ). Another study also showed that a carbohydrate food with higher GI values, such as bread, tended to be more strongly associated with colorectal cancer risk than a carbohydrate food with lower GI values such as pasta ${ }^{(40)}$. In the present study, cereal grains and vegetables and fruit were two main food sources of non-fibre carbohydrate. However, the proportion of food sources was different among different GI quartile groups. Compared with the first quartile of GI group, non-fibre carbohydrate of the last quartile of GI had a higher proportion of food sources from cereal grains, but a lower proportion from vegetables and fruit. It was reported that cereal grains consumed among Chinese were predominantly from white rice, which contained a high amount of refined carbohydrates and could provoke a rapid postprandial increase in blood glucose ${ }^{(41)}$. The modulation of insulin and IGF might be the main mechanism underlying the positive association between dietary GI and colorectal cancer risk. Long-term intake of a high-GI diet results in hyperglycaemia, and consequently hyperinsulinaemia ${ }^{(42)}$. Insulin can regulate gene expression and mitogenicity ${ }^{(43)}$, and enhance the concentration of IGF by inhibiting the level of IGF-binding proteins ${ }^{(8)}$ or by activating insulin/IGF receptors $^{(44)}$. IGF plays an important role in the development of colorectal cancer by inhibiting apoptosis and stimulating cell proliferation ${ }^{(9)}$.

Stratified analysis by sex showed that the inverse association between intakes of total carbohydrate, non-fibre carbohydrate and total fibre and the risk of colorectal cancer was observed among men but not among women. Similarly, the Prostate, Lung, Colorectal and Ovarian screening study conducted in the USA found that carbohydrate intake was inversely associated with colorectal advanced adenomas only in men but not in women. The adjusted OR was 0.74 (95\% CI 0.57, 0.95) and 0.87 (95\% CI $0.62,1.22)$, respectively ${ }^{(45)}$. The advanced adenomas included cancer in situ with high-grade dysplasia, which was a precancerous lesion of colorectal cancer. As presented in our study, the amount of carbohydrate and fibre intake among men was higher than that among women, which might partly explain the inverse association only observed in men. Moreover, the postprandial insulin response may be regulated by sex hormones. It was reported that girls demonstrate a greater blood insulin response than the boys after breakfast in a randomised controlled trail ${ }^{(46)}$. The sex influenced the metabolism of carbohydrate, such as women had less reliance on carbohydrate oxidation to ensure fuel requirements during exercises, which was mediated by oestrogen ${ }^{(47)}$. However, the evidence about the sex difference is still limited and it may be a chance finding in our study. Thus, whether the association between carbohydrate and fibre intake and colorectal cancer risk is modified by sex requires further confirmation.

Subgroup analysis by sources of controls showed that the associations between the intakes of non-fibre carbohydrate, fibre, starch, dietary GI and GL and colorectal cancer risk were similar when only one set of controls was used. However, the intake of total carbohydrate was inversely associated with colorectal cancer risk when only using community-derived controls. One possible explanation might be that communityderived controls consumed more total carbohydrate than hospital-derived controls. In the present study, the intake of total carbohydrate was 277.6 (SD 48.8) g/d among communityderived controls and 260.6 (sD 50.5) g/d among hospital-derived controls. Another reason might be that community-derived controls were engaged in more household and leisure-time activities than hospital-derived controls (43.3 MET-h/week for community-derived controls and 37.7 MET-h/week for hospitalderived controls). As people who are more active in physical activity might consume more carbohydrate ${ }^{(10)}$, it is possible that diet with high carbohydrate indicates a healthy lifestyle ${ }^{(48)}$.

Our study has the following strengths. First of all, a validated FFQ was used to assess the frequency and quantity of intakes of total carbohydrate, non-fibre carbohydrate, total fibre and starch. Second, comprehensive indicators were used to assess dietary carbohydrate intake and colorectal cancer risk in the present study. GI ranks the foods' carbohydrate quality based on their postprandial blood glucose effects, and GL further considers the quantity of carbohydrate consumed, which is a comprehensive measure of both the blood glucose effects (quality) of the food and the total quantity of carbohydrate consumed. Third, the GI values of most foods were from Chinese Food Composition Table, which could accurately reflect glucose response of local foods consumed by Chinese and offer an accurate and comprehensive estimate of dietary GI. Fourth, the potential confounders including dietary and non-dietary factors were adjusted for in the analysis. Moreover, the sample size was larger than that of most previous relevant case-control studies, so that we have enough power to detect small associations with colorectal cancer risk. Finally, this is the first study to detect association between dietary intake of nonfibre carbohydrate, starch and colorectal cancer risk among Chinese people.

Some potential limitations existed in the present study. First, selection bias may exist in hospital-based case-control studies. Even though the colorectal cancer patients were recruited from only one hospital, Sun Yat-sen University Cancer Center, it is the biggest cancer centre in Southern China. The colorectal cancer patients from this hospital shared similar clinical characteristics with those from other big hospitals in Guangdong or in mainland China ${ }^{(49)}$. Furthermore, the high participation rate (87.93\% for cases and $88.02 \%$ for hospital-derived controls) also helped to reduce selection bias in our study. Second, it is known that recall bias is difficult to rule out in case-control designed studies. To minimise this bias, we included only newly diagnosed cases and interviewed them as soon as possible after diagnosis. The average time interval between the diagnosis of colorectal cancer and study interview was $5.0 \mathrm{~d}$ for the case subjects. In addition, food photographs with usual portion sizes were also provided to the participants to help them better quantify their dietary intake. Third, random measurement error of diet is also of concern in the estimation of usual intake. As this misclassification bias is likely to be non-differential and lead the OR to the null, our results may tend to be conservative. Fourth, although we have tried our best 
to adjust a wide range of kinds of confounders in our analysis, some potential residual confounding factors may still remain. For example, information on drug usage was not collected which may influence the colorectal cancer risk. Fifth, the FFQ was not specifically developed to estimate dietary GI and GL, which may not accurately reflect the blood glucose response to mixed dishes compared with individual food items.

In conclusion, this study showed that dietary GI was positively associated with colorectal cancer risk. However, total carbohydrate, non-fibre carbohydrate intake, starch and dietary GL were not found to be related to an increased risk of colorectal cancer in a Chinese population.

\section{Acknowledgements}

The authors gratefully acknowledge the contribution of the study participants.

This study was supported by Guangdong Natural Science Foundation (nos: 2016A030313225, 2014A030313188). The funders had no role in the design, analysis or writing of this article.

The authors' responsibilities were as follows: J. H. conducted the data collection and data analysis, and writing of this paper. M. X., H. L., N.-Q. Z. and W.-Q. H. participated in data collection and data entry. Y.-J. F. and Z.-Z. P. contributed to the field work. Y.-M. C. analysed and interpreted the data. C.-X. Z. was responsible for designing and writing grants, supervision of the research and writing of this paper.

The authors declare that there are no conflicts of interest.

\section{References}

1. World Cancer Research Fund International \& American Institute for Cancer Research (2017) Continuous update project report: diet, nutrition, physical activity and colorectal cancer. wcrf.org/colorectal-cancer-2017 (accessed January 2018).

2. Chen W, Zheng R, Baade PD, et al. (2016) Cancer statistics in China, 2015. CA Cancer J Clin 66, 115-132.

3. Huxley RR, Ansary-Moghaddam A, Clifton P, et al. (2009) The impact of dietary and lifestyle risk factors on risk of colorectal cancer: a quantitative overview of the epidemiological evidence. Int J Cancer 125, 171-180.

4. Wiseman M (2008) The second World Cancer Research Fund/ American Institute for Cancer Research expert report. Food, nutrition, physical activity, and the prevention of cancer: a global perspective. Proc Nutr Soc 67, 253-256.

5. Deng L, Gui Z, Zhao L, et al. (2012) Diabetes mellitus and the incidence of colorectal cancer: an updated systematic review and meta-analysis. Dig Dis Sci 57, 1576-1585.

6. De Bruijn KM, Arends LR, Hansen BE, et al. (2013) Systematic review and meta-analysis of the association between diabetes mellitus and incidence and mortality in breast and colorectal cancer. Br J Surg 100, 1421-1429.

7. Giovannucci E, Harlan DM, Archer MC, et al. (2010) Diabetes and cancer: a consensus report. CA Cancer J Clin 60, 207-221.

8. Suikkari AM, Koivisto VA, Rutanen EM, et al. (1988) Insulin regulates the serum levels of low molecular weight insulin-like growth factor-binding protein. J Clin Endocrinol Metab 66, 266-272.

9. Kaaks R \& Lukanova A (2001) Energy balance and cancer: the role of insulin and insulin-like growth factor-I. Proc Nutr Soc 60, 91-106.
10. Greenwood DC, Threapleton DE, Evans CE, et al. (2013) Glycemic index, glycemic load, carbohydrates, and type 2 diabetes: systematic review and dose-response meta-analysis of prospective studies. Diabetes Care 36, 4166-4171.

11. Aune D, Chan DS, Lau R, et al. (2012) Carbohydrates, glycemic index, glycemic load, and colorectal cancer risk: a systematic review and meta-analysis of cohort studies. Cancer Causes Control 23, 521-535.

12. Jenkins DJ, Wolever TM, Taylor RH, et al. (1981) Glycemic index of foods: a physiological basis for carbohydrate exchange. Am J Clin Nutr 34, 362-366.

13. Du H, van der ADL, van Bakel MM, et al. (2008) Glycemic index and glycemic load in relation to food and nutrient intake and metabolic risk factors in a Dutch population. Am J Clin Nutr 87, 655-661.

14. Sieri S, Krogh V, Agnoli C, et al. (2015) Dietary glycemic index and glycemic load and risk of colorectal cancer: results from the EPIC-Italy study. Int J Cancer 136, 2923-2931.

15. Galeone C, Pelucchi C \& La Vecchia C (2012) Added sugar, glycemic index and load in colon cancer risk. Curr Opin Clin Nutr Metab Care 15, 368-373.

16. Choi Y, Giovannucci E \& Lee JE (2012) Glycaemic index and glycaemic load in relation to risk of diabetes-related cancers: a meta-analysis. Br J Nutr 108, 1934-1947.

17. Aune D, Chan DS, Lau R, et al. (2011) Dietary fibre, whole grains, and risk of colorectal cancer: systematic review and doseresponse meta-analysis of prospective studies. BMJ 343, d6617.

18. Higginbotham S, Zhang ZF, Lee IM, et al. (2004) Dietary glycemic load and risk of colorectal cancer in the Women's Health Study. J Natl Cancer Inst 96, 229-233.

19. Borugian MJ, Sheps SB, Whittemore AS, et al. (2002) Carbohydrates and colorectal cancer risk among Chinese in North America. Cancer Epidemiol Biomarkers Prev 11, 187-193.

20. Franceschi S, La Vecchia C, Russo A, et al. (1998) Macronutrient intake and risk of colorectal cancer in Italy. Int $J$ Cancer 76, 321-324.

21. Levi F, Pasche C, Lucchini F, et al. (2002) Macronutrients and colorectal cancer: a Swiss case-control study. Ann Oncol 13, 369-373.

22. Li HL, Yang G, Shu XO, et al. (2011) Dietary glycemic load and risk of colorectal cancer in Chinese women. Am J Clin Nutr 93, 101-107.

23. Zhong X, Fang YJ, Pan ZZ, et al. (2013) Dietary fat, fatty acid intakes and colorectal cancer risk in Chinese adults: a casecontrol study. Eur J Cancer Prev 22, 438-447.

24. World Health Organization (1998) Guidelines for Controlling and Monitoring the Tobacco Epidemic. Geneva: WHO.

25. Ainsworth BE, Haskell WL, Whitt MC, et al. (2000) Compendium of physical activities: an update of activity codes and MET intensities. Med Sci Sports Exerc 32, S498-S504.

26. Ainsworth BE, Haskell WL, Herrmann SD, et al. (2011) 2011 Compendium of Physical Activities: a second update of codes and MET values. Med Sci Sports Exerc 43, 1575-1581.

27. Zhang CX \& Ho SC (2009) Validity and reproducibility of a food frequency Questionnaire among Chinese women in Guangdong province. Asia Pac J Clin Nutr 18, $240-250$

28. Zhong X, Fang YJ, Pan ZZ, et al. (2014) Dietary fiber and fiber fraction intakes and colorectal cancer risk in Chinese adults. Nutr Cancer 66, 351-361.

29. Yang YX, Wang GY \& Pan XC (2002) China Food Composition. vol. 42. Beijing: Peking University Medical Press.

30. US Department of Agriculture (2012) USDA National Nutrient Database for Standard Reference, Release 25. https://ndb.nal. usda.gov/ndb/nutrients/index (accessed April 2017). 
31. George SM, Mayne ST, Leitzmann MF, et al. (2009) Dietary glycemic index, glycemic load, and risk of cancer: a prospective cohort study. Am J Epidemiol 169, 462-472.

32. Willett WC, Howe GR \& Kushi LH (1997) Adjustment for total energy intake in epidemiologic studies. Am J Clin Nutr 65 , 1220S-1228S 1229S-1231S.

33. Sieri S, Agnoli C, Pala V, et al. (2017) Dietary glycemic index, glycemic load, and cancer risk: results from the EPICItaly study. Sci Rep 7, 9757.

34. Leonel AJ \& Alvarez-Leite JI (2012) Butyrate: implications for intestinal function. Curr Opin Clin Nutr Metab Care 15, 474-479.

35. Higgins JA \& Brown IL (2013) Resistant starch: a promising dietary agent for the prevention/treatment of inflammatory bowel disease and bowel cancer. Curr Opin Gastroenterol 29, 190-194.

36. Peres J (2014) Resistant starch may reduce colon cancer risk from red meat. J Natl Cancer Inst 106, 6.

37. Xu M, Chen YM, Huang J, et al. (2016) Flavonoid intake from vegetables and fruits is inversely associated with colorectal cancer risk: a case-control study in China. Br J Nutr 116, 1275-1287.

38. Englyst HN, Kingman SM \& Cummings JH (1992) Classification and measurement of nutritionally important starch fractions. Eur J Clin Nutr 46, Suppl. 2, S33-S50.

39. Birt DF, Boylston T, Hendrich S, et al. (2013) Resistant starch: promise for improving human health. Adv Nutr 4, 587-601.

40. Augustin LS, Malerba S, Lugo A, et al. (2013) Associations of bread and pasta with the risk of cancer of the breast and colorectum. Ann Oncol 24, 3094-3099.

41. Yu D, Shu XO, Li H, et al. (2013) Dietary carbohydrates, refined grains, glycemic load, and risk of coronary heart disease in Chinese adults. Am J Epidemiol 178, 1542-1549.
42. Sacks FM, Carey VJ, Anderson CA, et al. (2014) Effects of high vs low glycemic index of dietary carbohydrate on cardiovascular disease risk factors and insulin sensitivity: the OmniCarb randomized clinical trial. JAMA $\mathbf{3 1 2}$, 2531-2541.

43. Burgering BM, Medema RH, Maassen JA, et al. (1991) Insulin stimulation of gene expression mediated by p21ras activation. Embo J 10, 1103-1109.

44. Rosenzweig SA \& Atreya HS (2010) Defining the pathway to insulin-like growth factor system targeting in cancer. Biochem Pharmacol 80, 1115-1124.

45. Flood A, Peters U, Jenkins DJ, et al. (2006) Carbohydrate, glycemic index, and glycemic load and colorectal adenomas in the Prostate, Lung, Colorectal, and Ovarian Screening Study. Am J Clin Nutr 84, 1184-1192.

46. Cooper SB, Dring KJ, Morris JG, et al. (2017) Sex differences in adolescents' glycaemic and insulinaemic responses to high and low glycaemic index breakfasts: a randomised control trial. Br J Nutr 117, 541-547.

47. Devries MC (2016) Sex-based differences in endurance exercise muscle metabolism: impact on exercise and nutritional strategies to optimize health and performance in women. Exp Physiol 101, 243-249.

48. Strayer L, Jacobs DJ, Schairer C, et al. (2007) Dietary carbohydrate, glycemic index, and glycemic load and the risk of colorectal cancer in the BCDDP cohort. Cancer Causes Control 18, 853-863.

49. Dai Z, Zheng RS, Zou XN, et al. (2012) Analysis and prediction of colorectal cancer incidence trend in China. Zhonghua Yu Fang Yi Xue Za Zhi 46, 598-603. 